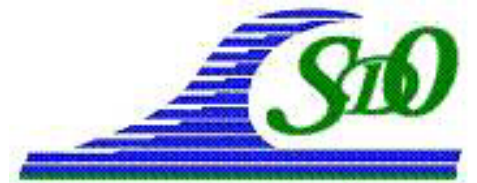

XI ${ }^{\text {èmes }}$ Journées Nationales Génie Côtier - Génie Civil

Les Sables d'Olonne, 22-25 juin 2010

DOI:10.5150/jngcgc.2010.060-L C Editions Paralia CFL

disponible en ligne - http://www.paralia.fr - available online

\title{
Profileurs acoustiques : un nouveau pas vers la connaissance des flux de polluants en assainissement urbain
}

\author{
Erwan LE BARBU ${ }^{1}$, Frédérique LARRARTE ${ }^{2}$
}

1. Laboratoire Régional des Ponts et Chaussées de Blois, 11 rue Laplace, CS 2912, 41029 Blois Cedex, France. erwan.le-barbu@developpement-durable.gouv.fr

2 Laboratoire Central des Ponts et Chaussées, Division eau et environnement, Route de Bouaye, BP 4129, 44341 Bouguenais Cedex, France.

frederique.larrarte@lcpc.fr

\section{Résumé :}

Depuis une dizaine d'année, les techniques hydro-acoustiques ont permis aux hydrographes marins puis fluviaux de se doter d'appareils de mesures de plus en plus rapides et précis dans la mesure des paramètres physiques du milieu aquatique. Ces techniques s'étendent actuellement au domaine des réseaux d'assainissement. En particulier, les profileurs ultrasonores permettent de réaliser des mesures sur une verticale, très rapidement, avec du matériel peu intrusif. De plus, ces appareils permettent théoriquement de mesurer simultanément la vitesse dans les 3 directions (longitudinales, transversales, verticales) et l'intensité rétrodiffusée. Dans le domaine de l'assainissement, une mesure conjointe des profils de vitesses et des concentrations en MES ne semble pas avoir été réalisée avec les appareils commercialisés. Cet article présente l'étude expérimentale effectuée afin de préciser les potentialités des profileurs acoustiques en vue de mesurer les flux de polluants en collecteurs d'assainissement. Après quelques rappels de théorie, les matériels mis en œuvre sont décrits. Les premiers résultats sont présentés et discutés. Enfin les pistes de travaux complémentaires sont exposées.

Mots-clés :

Profileur acoustique - Matières en suspension - Vitesse - Réseau d'assainissement

\section{Introduction}

Les techniques hydro-acoustiques, initialement développées pour des applications maritimes, se sont étendues au domaine fluvial et gagnent maintenant le domaine des réseaux d'assainissement et enfin le domaine des modèles réduits, permettant ainsi d'explorer des milieux de plus en plus contraints.

Ces outils, qui utilisent des ultrasons, sont des appareils de mesure aux potentialités nombreuses. Ils permettent notamment de réaliser des mesures sur une verticale (par pas d'espacement très faible), très rapidement, avec du matériel peu intrusif. De plus, ces appareils permettent théoriquement de mesurer simultanément la vitesse dans les 3 
directions (longitudinale, transversale, verticale) et l'intensité rétrodiffusée. Cette dernière donne des indications sur les caractéristiques des Matières en Suspension (MES) ou encore sur la nature du fond (fond dur, sableux ou vaseux). Toutefois, l'utilisation des profileurs nécessite une bonne compréhension des phénomènes physiques régissant la propagation des ondes acoustiques dans le milieu. Ainsi l'utilisation des profileurs acoustiques pour déterminer la concentration en matières en suspension reste pour le moment au stade expérimental que ce soit en laboratoire (THORNE et al., 1993 ; ADMIRAAL \& GARCIA, 2000; THORNE \& HANES, 2002), dans le domaine maritime (HOINTINK \& HOEKSTRA, 2005 ; TESSIER, 2006), fluvial (LE COZ et al., 2008; LE BARBU \& LE COZ, 2008) et en modèle physique réduit (BRICAULT, 2006). Dans le domaine de l'assainissement, une mesure conjointe des profils de vitesses et des concentrations en MES ne semble pas avoir été réalisée avec les appareils commercialisés. L'Institut de Mécanique des Fluides et des Structures de Strasbourg développe un prototype qui permettra de réaliser des mesures de profils des concentrations en matières en suspension (ABDA et al., 2008).

Cet article présente l'étude expérimentale effectuée afin de préciser les potentialités des profileurs acoustiques en vue de mesurer les flux de polluants en collecteurs d'assainissement. Après quelques rappels de théorie, les matériels mis en œuvre sont décrits. Les premiers résultats sont présentés et discutés. Enfin les pistes de travaux complémentaires sont exposées.

\section{Théorie}

Un capteur ultrasonore est muni d'un transducteur qui émet des ondes. Le signal acoustique frappe les cibles présentes dans le fluide. Il est alors renvoyé dans toutes les directions y compris celle du transducteur. Deux paramètres de l'onde ré-émise sont utiles afin de caractériser le milieu traversé :

- le changement de fréquence entre l'onde ré émise et l'onde incidente (appelé effet Doppler) traduit la vitesse des cibles,

- l'intensité du signal peut permettre de caractériser les cibles (quantité, forme, masse, ...).

L'analyse des décalages de fréquence est une technique éprouvée qui permet d'obtenir des profils de vitesse et est utilisé par les équipes de jaugeurs dans le milieu fluvial (LE COZ et al., 2008). Par contre, l'analyse des profils d'intensité en est au stade expérimental. Leur forme théorique est régie par l'équation du sonar. Elle permet d'évaluer les pertes d'intensité du signal lors de sa propagation dans la colonne d'eau et lors de sa ré-émission par les particules en suspension. La formule simplifiée de cette équation est la suivante (LURTON, 2002) :

$\mathrm{NR}=\mathrm{NE}+\mathrm{IC}-2 \mathrm{PT}$

où NR est l'intensité acoustique reçue par le transducteur $(\mathrm{dB})$, NE l'intensité acoustique émise par le transducteur et perçue à $1 \mathrm{~m}$ de celui ci $(\mathrm{dB})$, IC l'indice de 
cible (capacité des réflecteurs à ré-émettre l'onde ultra sonore) de l'ensemble des MES retro diffusant le signal (dB), PT les pertes par transmission lors de la propagation de l'onde acoustique (dB). Différents auteurs (THORNE \& HANES, 2002; TESSIER, 2006; HOITINK \& HOEKSTRA, 2005) s'accordent à penser que la nature des sédiments (organique ou minérale) est un facteur essentiel pour l'évaluation de IC qui peut être déterminé par l'équation (2) :

IC $=10 \log _{10}\left(\mathrm{C}_{\mathrm{m}}\right)+20 \log _{10}\left(\mathrm{f}\left(\rho_{\mathrm{p}} ; \mathrm{a} ; \mathrm{k} ; \mathrm{c} ; \ldots\right)\right)$

où $\mathrm{C}_{\mathrm{m}}$ est la concentration $\left(\mathrm{en} \mathrm{k} \mathrm{m}^{-3}\right), \rho_{\mathrm{p}}$ la masse volumique $\left(\mathrm{kg} \mathrm{m}^{-3}\right)$, a le rayon de la particule, $\mathrm{k}$ le nombre d'onde. La célérité des ultrasons $\mathrm{c}$ est fonction de la température de l'eau, de la salinité et de la profondeur (COPPENS, 1981). Pour les eaux usées, l'influence des deux derniers paramètres est négligeable. A $20^{\circ} \mathrm{C}$, la célérité est égale à $1480 \mathrm{~m} \mathrm{~s}^{-1}$. THORNE et al. (1993) ont proposé l'équation (3) pour quantifier l'atténuation :

$\mathrm{PT}=10 \log _{10}(\psi \mathrm{R})+\alpha \mathrm{R}$

Où $\psi$ est la dispersion cylindrique près de l'émetteur (distance de Fresnel), $\alpha$ l'atténuation telle que $\alpha=\alpha_{\mathrm{w}}+\alpha_{\mathrm{p}}$ avec $\alpha_{\mathrm{w}}$ l'atténuation due au milieu continu (ici l'eau) et $\alpha_{\mathrm{p}}$ celle due aux cibles.

(FISHER \& SIMMONS, 1977) ont proposé une loi pour calculer $\alpha_{\mathrm{w}}$ en milieu marin. (ADMIRAAL \& GARCIA, 2000) ont adapté cette expression pour les écoulements fluviaux (sans prise en compte de la salinité ni de la pression) :

$\alpha_{\mathrm{w}} \approx\left(55,9-2,37 \mathrm{~T}+0,05 \times \mathrm{T}^{2}\right) 10^{-15} \mathrm{f}_{\mathrm{o}}{ }^{2}(\mathrm{en} \mathrm{Pa} / \mathrm{m})$

où $\mathrm{T}$ est la température de l'eau $\left({ }^{\circ} \mathrm{C}\right)$ et $\mathrm{f}_{\mathrm{o}}$ la fréquence du signal émis $(\mathrm{Hz})$.

L'atténuation $\alpha_{\mathrm{p}}$ dépend de la viscosité pour les particules de petite taille (URICK, 1948) alors qu'elle est associée à des phénomènes de diffusion pour les particules plus grandes (SHENG \& HAY, 1988; THORNE et al., 1993). Après lissage de données expérimentales, (THORNE \& HANES, 2002) ont proposé la relation (5) pour des particules rigides et sphériques de sable

$\alpha_{\mathrm{p}}=\frac{1,1 \mathrm{k}_{\alpha} \mathrm{x}^{4}}{1+1,3 \mathrm{x}^{2}+4 / 3 \mathrm{k}_{\alpha} \mathrm{x}^{4}} \frac{\mathrm{C}_{\mathrm{m}}}{\text { a. } \rho_{\mathrm{p}}}$

où $\mathrm{k}_{\alpha}=0,18$ et $\mathrm{x}=2 \pi \mathrm{a} / \lambda$, où $\lambda$ est la longueur d'onde. Bien que les particules en suspension dans les eaux usées ne soient pas du sable, cette expression permet d'estimer l'ordre de grandeur du coefficient d'atténuation. Une étude expérimentale (LARRARTE \& FRANÇOIS, 2008) a montré que l'atténuation des ultrasons par les particules en suspension dans les eaux usées est négligeable pour les concentrations usuelles.

\section{Etude expérimentale}

Le profileur PC-ADP a attiré notre attention dans le cadre de l'étude des distributions spatiales des vitesses dans un collecteur (LARRARTE \& COTTINEAU, 2008). En effet, il doit permettre : 
- d'acquérir des informations sur un profil vertical en un temps très bref ;

- d'obtenir des mesures près du fond.

Le capteur utilisé est un PC-ADP (Pulse Coherent - Acoustic Doppler Profiler) fabriqué par la firme américaine SonTek. Il convient toutefois de noter que l'appareil utilisé dans cette étude est un quasi-prototype acquis dans une optique de recherche et non à des fins de gestion ou de suivi en continu, il n'a donc fait l'objet d'aucune démarche de miniaturisation ou de portabilité. Le matériel acquis est une évolution "shallow water" (faibles hauteurs d'eau) du modèle usuellement proposé par le fabricant. Cela se traduit par une bande morte ("blank") de 0,20 m et un maximum de 100 cellules, chaque cellule ayant une hauteur minimale de $0,02 \mathrm{~m}$. La fréquence des mesures est un paramètre modifiable. Il s'est avéré qu'à partir d'une mesure toutes les 2 secondes, la qualité de la mesure n'était plus impactée. Dans un souci de qualité et de rapidité de la campagne de mesure et de quantité de données à gérer, le pas de temps de 5 secondes a été choisi.

Le PC-ADP émet trois faisceaux ultrasonores qui permettent de calculer les 3 composantes du champ de vitesses. L'analyse du décalage de fréquence entre l'onde émise $(1500 \mathrm{kHz})$ et l'onde ré-émise par les MES permet de déterminer par effet Doppler la vitesse des particules présentes dans la colonne d'eau. Un maillage temporel de l'analyse du signal ré-émis permet de déterminer la valeur de la vitesse des MES sur un maillage vertical. Par ailleurs, la célérité des ondes dans l'eau permet de réaliser des mesures instantanées et de les répéter très rapidement (jusqu'à une demi-seconde d'intervalle). La vitesse des particules est assimilée à celle de l'eau et donc ainsi la vitesse de l'écoulement est mesurée. Du fait de l'inclinaison de $15^{\circ}$ des transducteurs avec la verticale, chacun des trois volumes de mesure correspondant à la cellule i située à la profondeur di se trouve à une distance horizontale :

$\Delta=\mathrm{d}_{\mathrm{i}} \tan \left(15^{\circ}\right)$

de l'axe vertical du profileur. Par exemple, à $1 \mathrm{~m}$ de profondeur, $\Delta=0,52 \mathrm{~m}$. Les vitesses indiquées par le PC-ADP pour chaque cellule sont donc calculées à partir des vitesses radiales mesurées à l'intérieur de trois volumes dont l'écartement croît avec la profondeur. Compte tenu des dimensions du collecteur, les faisceaux couvrent une large fraction transversale de la section de mesure. Les profils des vitesses transversales et verticales correspondent en effet à un étalement spatial pouvant représenter plus du quart de la section transversale, ils ne sont donc pas exploités.

Le PC-ADP a été conçu pour des applications maritimes et mesure du haut vers le bas. Une fraction du signal émis se propage verticalement et est réfléchie par le fond avant les faisceaux qui se sont propagés selon un axe angulé de $15^{\circ}$ avec la verticale. Cela génère des lobes secondaires sur une hauteur $\mathrm{h}_{\mathrm{b}}$ (SIMPSON, 2001 ; LE COZ et al., 2008) sur laquelle les mesures sont biaisées. Cette hauteur peut être calculée par la relation :

$\mathrm{h}_{\mathrm{b}}=1-\cos \left(15^{\circ}\right)=0,03 \mathrm{~h}$ 


\section{Site expérimental}

Le site de mesure est situé dans le réseau unitaire de la ville de Nantes. Il s'agit d'un collecteur ovoïde à banquette de 2,3 m de hauteur maximale et de $1,5 \mathrm{~m}$ de largeur maximale. Le collecteur est en béton de bonne qualité avec un coefficient de Manning Strickler évalué à 70. La banquette est constituée d'une marche horizontale de $40 \mathrm{~cm}$ de large située à droite quand on regarde vers l'amont. Cette banquette représente $27 \%$ de la largeur maximale du collecteur. Le PC-ADP a été fixé sur un dispositif appelé Hydre (figure 1) dont le degré de liberté vertical a été utilisé pour amener le PC-ADP au contact de la surface libre. Cet échantillonneur bidimensionnel permet de réaliser des mesures de vitesses de l'écoulement et des prélèvements d'effluent sur un maillage de points couvrant l'ensemble de la section mouillée (LARRARTE \& COTTINEAU, 2008). En effet, dans un collecteur, la hauteur d'eau est variable selon l'heure de la journée et les évènements pluvieux. Des mesures avec le profileur ont été effectuées et comparées aux mesures de vitesses et de concentration en MES réalisées avec Hydre.

\section{Résultats}

Des mesures ont été effectuées pour des contextes pluviométriques variées. La figure 2 montre les profils de vitesses présentés en hauteur depuis le radier (situé en $\mathrm{z}=0$ ) comme usuellement en assainissement. Il s'agit de profils de temps de pluie obtenus le 24 avril 2008. On constate une très bonne concordance des profils obtenus avec le PC-ADP avec ceux obtenus avec Hydre sur plus de $60 \%$ de la hauteur. Les profils obtenus avec Hydre présentent une vitesse maximale en dessous de la surface libre, ce qui est caractéristique des écoulements étroits. Cela ne se voit pas sur les profils avec le PC-ADP car la bande morte ne permet pas d'effectuer de mesures dans les vingt premiers centimètres sous la tête. Il convient de signaler que le profileur permet d'effectuer toutes les mesures en moins d'une minute alors qu'il faut de 15 à 30 minutes pour acquérir un profil avec Hydre. De plus, on voit très nettement le décrochage du profil de vitesses obtenus avec le profileur lorsque les ultrasons rencontrent le béton du radier. Cela permet de définir la localisation du fond, lors de l'analyse des profils, avec une incertitude égale à la taille d'une cellule (soit ici $2 \mathrm{~cm}$ ).

La figure 3 montre une comparaison de l'intensité rétrodiffusée et de la concentration en matières en suspension. On note que, sur les deux profils, l'intensité décroît de la surface jusqu'à $0,1 \mathrm{~m}$ du fond puis augmente brusquement. Dans le même temps la concentration présente des fluctuations mais pas de gradient sur la verticale. Le taux de matière organique reste constant malgré des concentrations $70 \%$ plus élevées en novembre qu'en février. Ceci illustre le fait que la relation entre l'intensité rétrodiffusée et les caractéristiques des particules dépend de diverses caractéristiques. Dans l'état actuel des connaissances, l'expérimentateur peut utiliser la donnée d'intensité en ellemême mais sa traduction en concentration nécessite encore des mises au point tant au laboratoire que sur le terrain afin de déterminer si une relation de conversion peut-être 
Thème 3 - Instrumentation, mesures, imagerie et télédétection

trouvée et avec quelles hypothèses et limites d'utilisation. On pourra alors ensuite envisager de définir une procédure d'étalonnage.

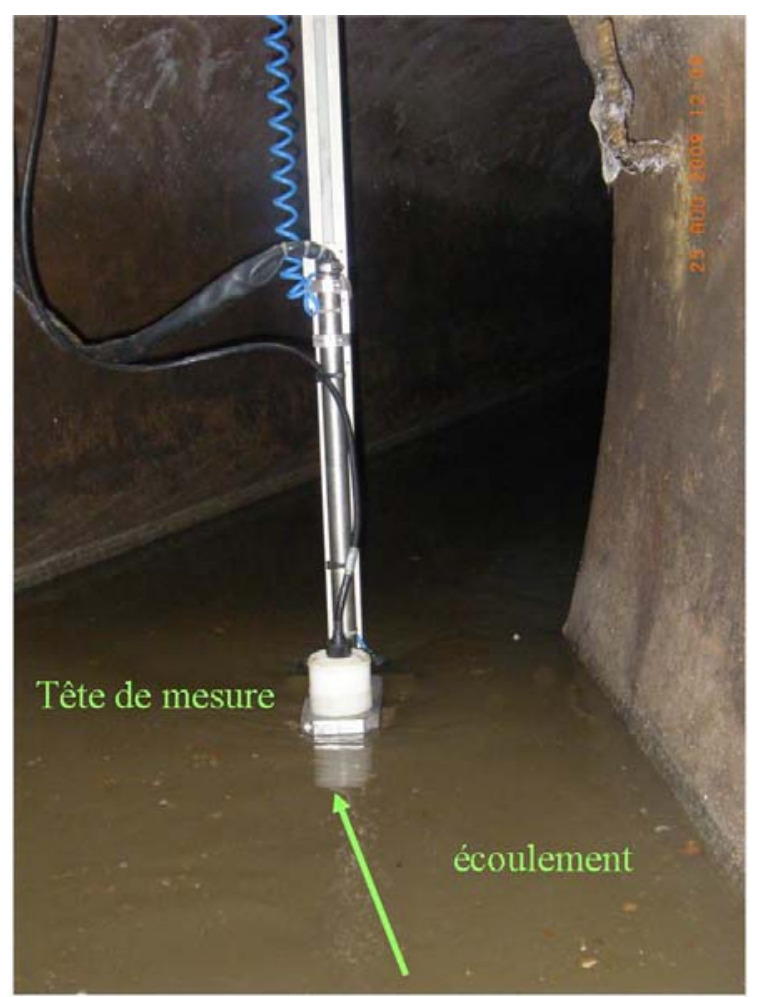

Figure 1. PC-ADP fixé sur Hydre.

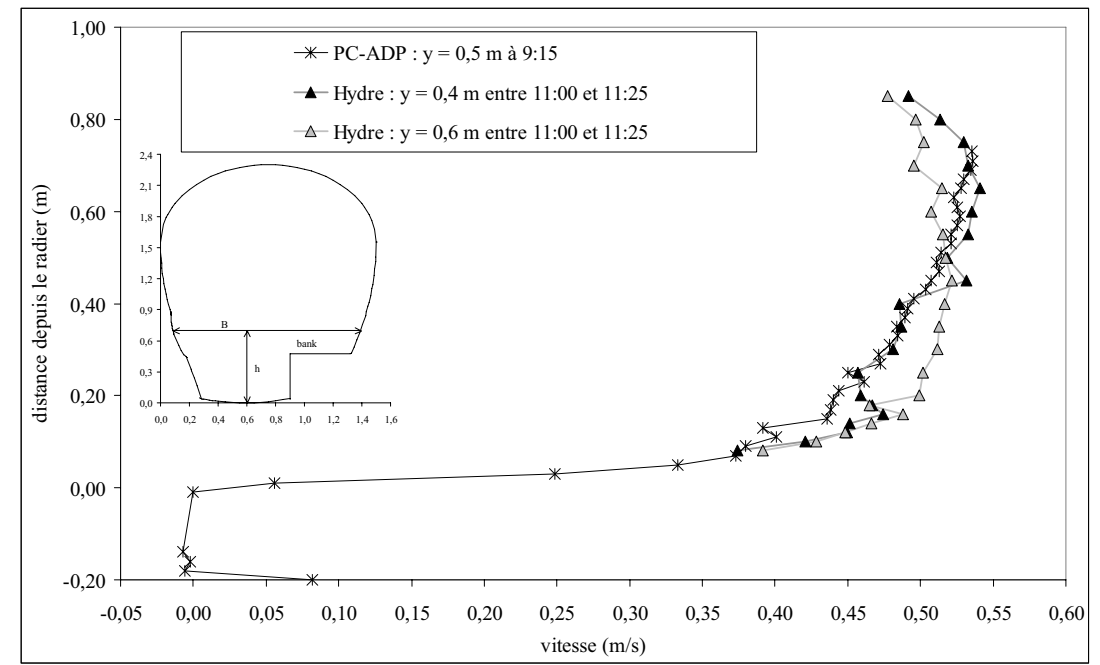

Figure 2. Comparaison des profils obtenus avec Hydre et le PC-ADP.

\section{Conclusion}

Cet article présente l'étude effectuée afin de préciser les potentialités des profileurs acoustiques pour mesurer les flux polluants en collecteurs d'assainissement. Un 
profileur a été mis en œuvre dans un collecteur unitaire et les résultats comparés à ceux obtenus avec un échantillonneur permettant de mesurer les vitesses dans la direction principales de l'écoulement et les concentrations en matières en suspension.

Les résultats montrent que le profileur permet de déterminer les profils de vitesse et notamment l'acquisition de données à proximité du fond, zone où les mesures sont extrêmement délicates avec les techniques usuelles du fait de la taille des capteurs, même si celles-ci sont fortement bruitées par les lobes secondaires. A contrario, le profileur ne permet pas de mesurer les vitesses près de la surface du fait de sa bande morte. Une modification du montage qui permettrait de décaler le profileur est à l'étude afin de s'affranchir de ce problème. L'utilisation des profils d'intensité pour déterminer les concentrations en matières en suspension en est au stade de la recherche. Ces travaux doivent se poursuivre afin de préciser l'influence de la température des effluents, de la concentration mais également des caractéristiques en particules.

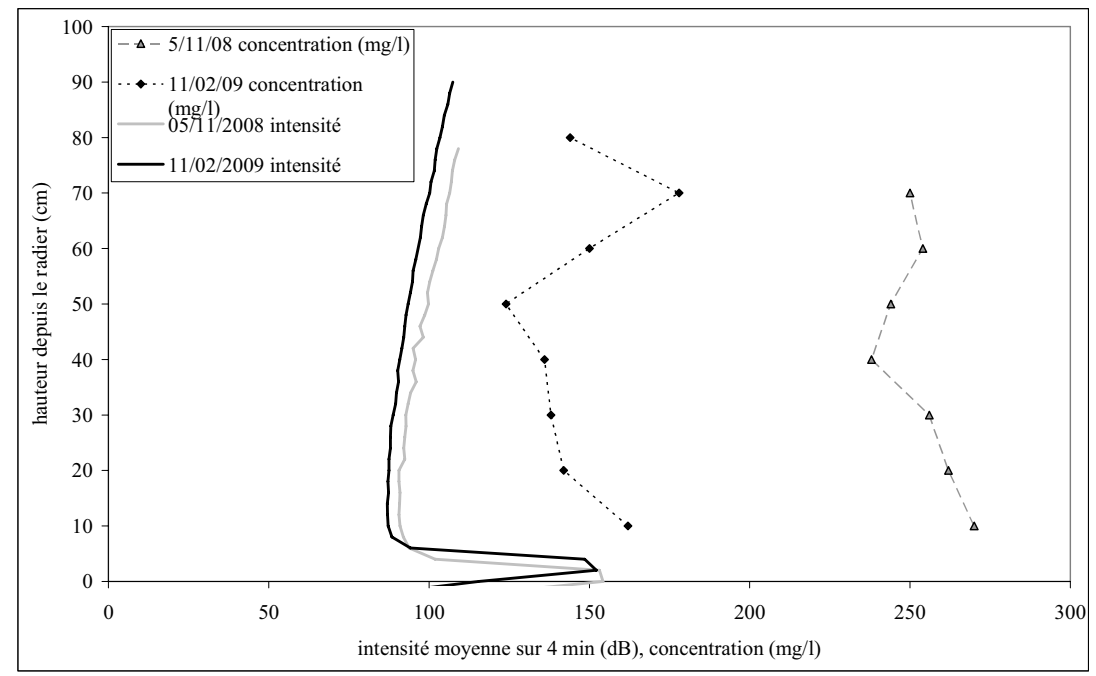

Figure 3. Profils d'atténuation et de concentrations en matières en suspension.

\section{Remerciements}

Les auteurs souhaitent remercier les agents de la division Eau et Environnement et le personnel de la Direction de l'Assainissement de Nantes Métropole pour leur soutien lors des expérimentations.

\section{Références}

ABDA F., AZBAID A., ENSMINGER D., FISHER S., FRANÇOIS P., SCHMITT P., PALLARES A. (2008). Ultrasonic device for real time sewage velocity and suspended particles concentration measurements. $11^{\text {th }}$ International Conference on Urban Drainage, Scotland, UK, 8 p. 
Thème 3 - Instrumentation, mesures, imagerie et télédétection

ADMIRAAL D.M., GARCIA M.H. (2000). Laboratory measurement of suspended concentration using an Acoustic Concentration Profiler (ACP). Experiments in fluids, Vol. 28, n² 2, 2000, pp 116-127.

BRICAULT M. (2006). Rétrodiffusion acoustique par une suspension en milieu turbulent : application à la mesure de profils de concentration pour l'étude de processus hydrosédimentaires. Thèse, INP Grenoble, $232 \mathrm{p}$.

COPPENS A.B. (1981). Simple equations for the speed of sound in Neptunian waters.

J. Acoust. Soc. Am. 69(3), pp 862-863. doi:10.1121/1.385486

FISHER F.H., SIMMONS V.P. (1977). Sound absorption in sea water. J Acoust Soc Am 62, pp 558-564. doi:10.1121/1.381574

HOITINK A.J.F., HOEKSTRA P. (2005). Observation of suspended sediment from $A D C P$ ans $O B S$ measurements in a mud dominated environnement. Coastal Engineering, 52(2), pp 103-118. doi:10.1016/j.coastaleng.2004.09.005

LARRARTE F., COTTINEAU L.-M. (2008). Le projet Hydre : échantillonnage 2D des vitesses et des concentrations en collecteur d'assainissement. Bulletin des Laboratoires des Ponts et Chaussées, $\mathrm{N}^{\circ}$. 272, pp 21-32.

LARRARTE F., FRANÇOIS P. (2008). Suspended solids and attenuation of ultrasonic beam. 11th International Conference on Urban Drainage, Edinburgh, Scotland, UK, 8 p. LE BARBU E., LE COZ J. (2008). Mesure hydro acoustique des flux de matières en suspension en rivière. Rapport de fin d'étude, année spéciale de l'Ecole Nationale Supérieure d'Hydraulique et de Mécanique de Grenoble, 55 p.

LE COZ J., PIERREFEU G., SAYSSET G., BROCHOT J.-F., MARCHAND P. (2008). Mesures hydrologiques par profileur Doppler - Guide pratique. Editions QUAE, 192 p. LURTON X. (2002). An Introduction to underwater acoustic. Springer-Verlag Principles and Applications, $347 \mathrm{p}$.

SHENG J., HAY A.E. (1988). An examination of the spherical scatterer approximation in aqueous suspensions of sand. J. Acoust. Soc. of Am, 83, pp 598-610. doi:10.1121/1.396153

SIMPSON M.R, (2001), Discharge Measurements Using a Broad-Band Acoustic Doppler Current Profiler, United States Geological Survey Open File Report01-1

THORNE P.D., HANES D.M. (2002). A review of acoustic measurement of small-scale sediment processes. Contiental Shelf Research, Vol. 22, $\mathrm{n}^{\circ}$ 4, pp 603-632. doi:10.1016/S0278-4343(01)00101-7

THORNE P.D., HARDCASTLE P.J., SOULSBY R.L. (1993). Analysis of acoustic measurements of suspended sediments, Journal of Geophysical Research, 98(C1), pp 899-910. doi:10.1029/92JC01855

TESSIER C. (2006). Caractérisation et dynamique des turbidités en zone côtières: l'exemple de la région marine Bretagne Sud. Thèse, Université de Bordeaux 1, 428 p.

URICK R.J. (1948). The absorption of sound in suspensions of irregular particles. J. Acoust. Soc. of Am., 20, pp 283-289. doi:10.1121/1.1906373 